\title{
INDICADOR DE AFECTACIÓN POR EVENTOS NATURALES EN EL CANTÓN DE ALAJUELA, Y SU RELACIÓN CON LA VULNERABILIDAD COMUNAL
}

\author{
AFFECTATION INDICATOR FOR NATURAL EVENTS \\ IN THE CANTÓN OF ALAJUELA, AND ITS RELATION \\ WITH THE COMMUNITY VULNERABILITY
}

\author{
Gustavo Barrantes-Castillo ${ }^{1}$ \\ Adolfo Quesada-Román ${ }^{2}$ \\ Daniela Campos-Durán ${ }^{3}$ \\ Katherine Padilla-Umaña ${ }^{4}$ \\ Universidad Nacional de Costa Rica, Costa Rica
}

http://dx.doi.org/10.15359/rgac.3-59.6

\section{RESUMEN}

Se construyó un indicador de afectación por amenazas naturales que considera la afectación directa e indirecta con base en las variables incluidas en la base de datos DesInventar y reportes de la CNE para el periodo 1970 - 2015 a nivel distrital. Dicha información permitió obtener la ubicación aproximada de las comunidades que han presentado la mayor afectación por eventos naturales.

\footnotetext{
1 Escuela de Ciencias Geográficas, Universidad Nacional de Costa Rica, Costa Rica. Correo electrónico: gbarrantes@una.cr

2 Escuela de Ciencias Geográficas, Universidad Nacional de Costa Rica, Costa Rica. Correo electrónico: adolfo.quesada@gmail.com

3 Observatorio Vulcanológico y Sismológico de Costa Rica, Universidad Nacional de Costa Rica, Costa. Rica. Correo electrónico: daniela.campos.duran@una.cr

4 Consultora Independiente, Costa Rica. Correo electrónico: kathy.p28@hotmail.com
}

Fecha de recepción: 17 de enero de 2017

Fecha de aceptación: 19 de abril de 2017 
Gustavo Barrantes-Castillo, Adolfo Quesada-Román, Daniela Campos-Durán,

Katherine Padilla-Umaña. Affectation indicator for natural events in the cantón of

Alajuela, and its relation with the community vulnerability

Asimismo, mediante una encuesta a las Asociaciones de Desarrollo Integral y posterior trabajo de campo se logró detallar las condiciones de vulnerabilidad de estas comunidades. Como resultado se identificaron los distritos con mayor afectación por amenazas naturales del cantón de Alajuela, así como las comunidades con mayor afectación histórica, por ende, con mayores condiciones de vulnerabilidad. Esta información será utilizada por la municipalidad de Alajuela para mitigación, prevención y atención de emergencias.

Palabras clave: Amenazas naturales, vulnerabilidad, gestión del riesgo, desastres, municipalidad

\begin{abstract}
An affectation indicator for natural hazards was created. This indicator considers direct and indirect affectation based on variables included in the DesInventar database and the CNE reports between 1970 and 2015 at a district level. This information led to determine the location of the most vulnerable communities of the Alajuela canton. After this process and with the cooperation of Integral Development Associations through interviews and fieldwork, the vulnerability of these populations was identified. As a result, the most affected districts and the most vulnerable communities to natural hazards in the Alajuela canton were identified. This information will be used by the Alajuela Municipality for risk mitigation, prevention, and management.
\end{abstract}

Keywords: Natural hazards, vulnerability, risk assessment, disasters, municipality

\title{
1. Introducción
}

La ubicación ístmica y tectónica de Costa Rica en un área de subducción provoca que el país presente una alta sismicidad, así como una actividad volcánica activa. Asimismo, su localización en la Zona Intertropical propicia la generación de tormentas, ciclones tropicales y frentes fríos, eventos que asociados a la topografía son generadores de deslizamientos. Esto sumado al crecimiento de la población, especialmente, en áreas de riesgo, y la falta de planificación territorial conllevan al aumento constante de personas, infraestructura y bienes que son afectados por estos eventos naturales. Por su parte, el Valle Central de Costa Rica, localizado en el centro del país, alberga cerca del $60 \%$ de la población nacional. De acuerdo con la base de datos de desastres DesInventar, las principales amenazas naturales que han generado afectación en los últimos 30 años en esta región son las inundaciones y los deslizamientos, los cuales representan el $57 \%$ y el $31 \%$, respectivamente, de todos los eventos reportados (LA RED, 2015).

Propiamente en el cantón de Alajuela, las condiciones geodinámicas, fisiográficas y sociales, lo hacen susceptible a la ocurrencia de desastres inducidos por eventos naturales, tales como sismicidad, vulcanismo, fuertes lluvias, inundaciones y deslizamientos. Cabe señalar que para el 
periodo 1984 - 2011 la población urbana del cantón paso de un 43,7\% a un $88 \%$, es decir tuvo un incremento poblacional del 22,5\% (CCP-UCR, 2014). Este aumento se asocia con una expansión del área urbana de grupos poblacionales que se asientan en áreas de riesgo, tal como la rivera de los ríos o terrenos de topografía irregular, alterando los sistemas naturales, sumado a ello se da un aumento de aguas de escorrentía y residuales, así como el inadecuado manejo de los desechos sólidos.

Ante este panorama y en relación con la Ley Nacional de Emergencias y Prevención del Riesgo No. 8488, en su artículo 27 señala: "En los presupuestos de cada institución pública, deberá incluirse la asignación de recursos para el control del riesgo de los desastres, considerando la prevención como un concepto afín con las prácticas de desarrollo que se promueven y realizan" (s. p.).

En concordancia con lo citado anteriormente, la Municipalidad de Alajuela solicitó un estudio para conocer el tipo de eventos naturales que han afectado históricamente al cantón y su distribución territorial, con la finalidad de planificar las acciones y presupuestos requeridos para disminuir las condiciones de riesgo en su cantón. Este propósito origina el presente estudio, el cual muestra la ubicación y caracterización de los sitios que se han visto afectados por eventos naturales, así como aquellos en condiciones de riesgo.

\section{Afectación por eventos naturales en el Valle Central periodo 1985 - 2013}

De acuerdo con la base de datos de DesInventar, entre 1985 y 2013 , en el Valle Central se contabilizaron 6705 reportes por afectaciones, los cuales se dieron por distintos tipos de eventos naturales, como se muestran en el cuadro 1 . 
Gustavo Barrantes-Castillo, Adolfo Quesada-Román, Daniela Campos-Durán,

Katherine Padilla-Umaña. Affectation indicator for natural events in the cantón of

Alajuela, and its relation with the community vulnerability

Cuadro 1. Tipos de amenazas naturales y número de eventos en el Valle Central, periodo 1985-2013.

\begin{tabular}{|l|l|}
\hline \multicolumn{1}{|c|}{ Tipo de amenaza } & Número de eventos \\
\hline Actividad Volcánica & 19 \\
\hline Avenida torrencial & 64 \\
\hline Deslizamiento & 2082 \\
\hline Inundación, lluvias, tempestad, tormenta eléctrica & 3829 \\
\hline Sequía & 5 \\
\hline Sismo & 143 \\
\hline Vendaval & 563 \\
\hline Total & $\mathbf{6 7 0 5}$ \\
\hline
\end{tabular}

Fuente: LA RED, 2015.

Como se aprecia en el cuadro anterior, los deslizamientos y las inundaciones son los eventos que presentan una mayor recurrencia de afectaciones en el Valle Central, cabe señalar que ambos representan el 88\% de los incidentes registrados para este periodo. Por su parte, los cantones más afectados fueron Desamparados con 737 eventos, San José con 574, Alajuela con 572, Cartago con 379 y Aserrí con 300, tal como se muestra en la siguiente figura 1.

162 Revista Geográfica de América Central No 59 ISSN 1011-484X, julio-diciembre 2017, pp. 159-196 
Figura 1. Cantones con mayor cantidad de reportes de afectaciones por eventos naturales en el Valle Central, periodo 1985 - 2013

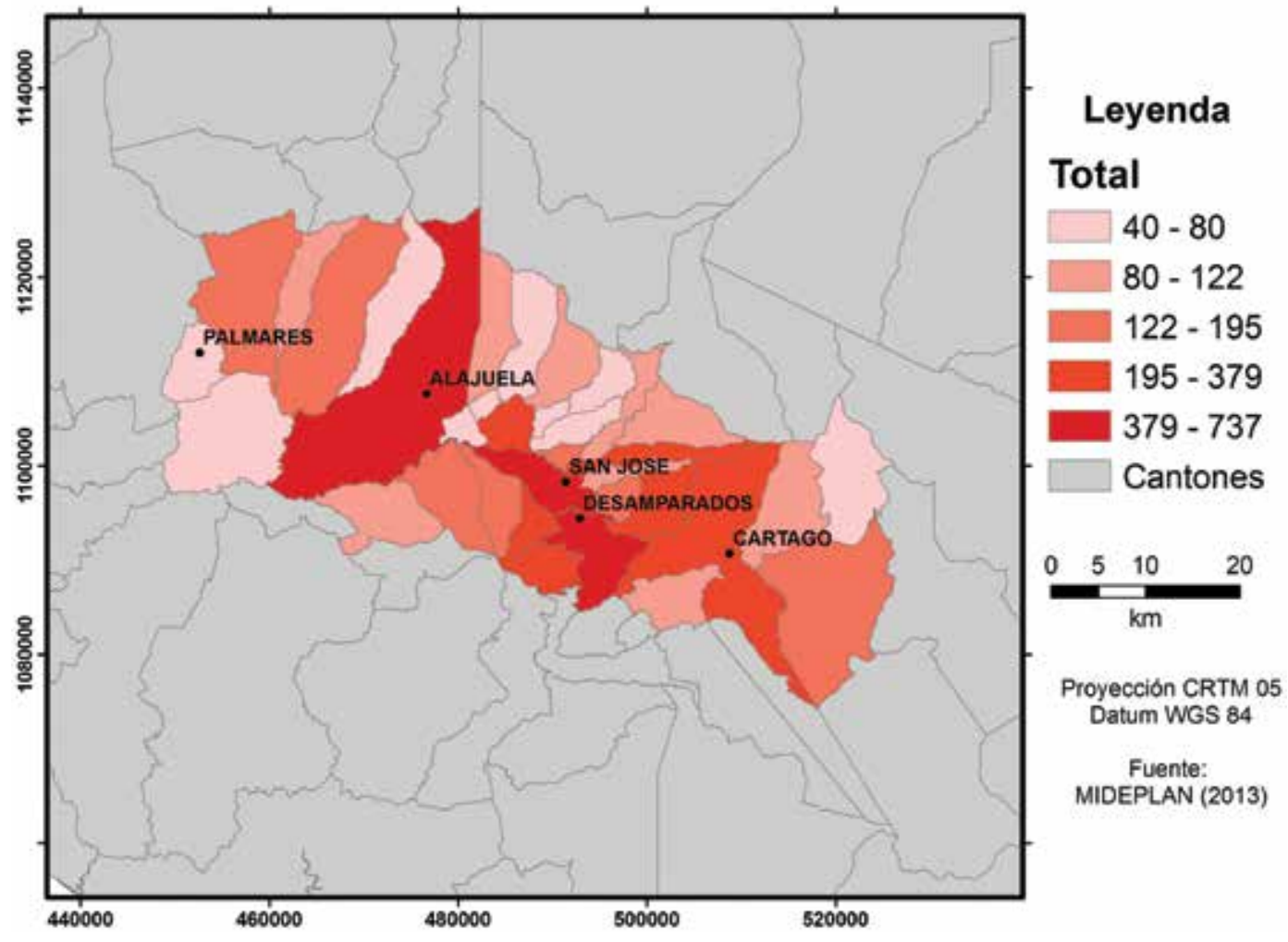

Fuente: LA RED, 2015.

De acuerdo con la Figura 1, se puede apreciar que entre los cantones que conforman el Valle Central, el cantón de Alajuela presentó entre 380 y 737 reportes de eventos entre 1985 y el 2013, encontrándose así, entre los tres cantones más afectados para este periodo.

Barrantes \& Malavassi (2015) señalan que el cantón de Alajuela, en las laderas SW del volcán Poás, puede verse afectado por caída de ceniza y gases volcánicos, así mismo la CNE (2015) advierte sobre la posibilidad de ocurrencia de lahares, específicamente, en los ríos Ciruelas, Alajuela, Itiquís, Tambor y Poás.

Además, la proximidad con fallas, como las de Viejo-Aguas Zarcas, El Ángel: Alajuela, Sabanilla, San Antonio-Picagres y la falla Víbora, lo hacen 
Gustavo Barrantes-Castillo, Adolfo Quesada-Román, Daniela Campos-Durán,

Katherine Padilla-Umaña. Affectation indicator for natural events in the cantón of

Alajuela, and its relation with the community vulnerability

susceptible a la actividad sísmica (Barrantes \& Salcedo, 2016). Ejemplo de ello, es el terremoto de Cinchona, ocurrido el 8 de enero de 2009, cuyo epicentro se localizó en Varablanca de Heredia y su magnitud fue de 6,2Mw con una profundidad de 4,6 km (Red Sismológica Nacional, 2009).

Este sismo de acuerdo con la Comisión Nacional de Emergencias (2009), provocó importantes daños en el cantón de Alajuela, específicamente en la comunidad de Poasito en el distrito de Sabanilla y las comunidades de Dulce Nombre y Fraijanes, en el distrito de San Isidro, donde se vieron afectadas 514 familias debido a daños y colapsos en viviendas, así como cortes en el suministro de los servicios de electricidad y agua potable, lo que llevo a declarar alerta roja en el cantón por 96 horas luego de ocurrido el sismo.

El cantón, además, se ha visto afectado por sistemas ciclónicos, como tormentas tropicales provenientes del Caribe y frentes fríos (Alfaro et al., 2010). Cabe señalar que por las diferencias de altitud las mayores precipitaciones se concentran en la zona montañosa de Alajuela, sobre terrenos de fuerte pendiente y ríos con profundos valles o cañones. Una vez que se desciende ladera abajo hacia las áreas de topografía relativamente plana y de mayor concentración poblacional del cantón, tal como los distritos de Alajuela, San José, San Isidro, San Antonio y Sabanilla, el cambio de pendiente y la intensidad de los procesos exógenos (meteorización, precipitación) y la alta concentración urbana favorecen la ocurrencia de deslizamientos e inundaciones. Estas últimas, representaron para el periodo 1985 - 2015 el $67 \%$ del total de eventos registrados para el municipio, mientras que los deslizamientos corresponden al 21\% (Figura 2). 
Figura 2. Número de reportes por tipo de amenazas naturales en el cantón de Alajuela, periodo 1985 y 2015

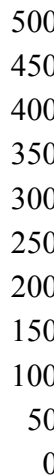

500

450

400

350

300

250

200

150

100

50
0
432

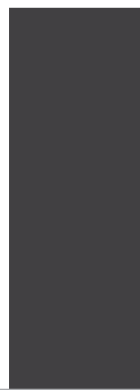

Inundaciones

Actividad volcánica

- Número de eventos

Fuente: LA RED, 2015.

\section{Metodología}

La primera etapa consistió en la elaboración de un indicador de afectación por amenazas naturales a nivel distrital en el cantón de Alajuela a partir de los registros de DesInventar (LA RED, 2015). En su construcción se utilizaron nueve variables, contenidas en la base de datos en mención (Cuadro 2), cuyo registro de eventos que han afectado al cantón de Alajuela se presentan para el periodo 1970 - 2015, dicha información se sistematizó y georreferenció mediante el uso de un Sistema de Información Geográfica (SIG). 
Gustavo Barrantes-Castillo, Adolfo Quesada-Román, Daniela Campos-Durán,

Katherine Padilla-Umaña. Affectation indicator for natural events in the cantón of

Alajuela, and its relation with the community vulnerability

Cuadro 2. Variables utilizadas para generar el indicador de afectación por amenazas naturales

\begin{tabular}{|l|c|}
\hline \multicolumn{1}{|c|}{ Variables } & \multicolumn{1}{c|}{ Indicador } \\
\hline Muertos & Afectación directa las personas \\
Desaparecidos & \\
Heridos & \\
\hline Damnificados & Afectación indirecta las personas \\
Afectados & \\
Evacuados & \\
Reubicados & \\
Viviendas destruidas & \\
Viviendas afectadas & \\
\hline
\end{tabular}

Fuente: Elaboración propia a partir de DesInventar, 2016.

En esta etapa, el primer paso, consistió en ubicar espacialmente los registros de DesIventar, esto se realizó de manera aproximativa, ya que dichos datos incluyen el cantón, distrito y solo en algunos casos hacen indicación al barrio donde se dio el incidente. El criterio de aproximación utilizado fue la relación entre el tipo de evento, las condiciones del relieve y la hidrografía del área. La imprecisión en la localización no se considera una limitante en el cálculo de indicador distrital de incidencia por desastres, ya que el distrito si está reportado.

El segundo pasó, consistió en el cálculo de un indicador de afectación por tipo evento natural, el algoritmo utilizado se detalla a continuación [Ec 1]:

$$
\mathrm{IA}=\mathrm{AD}+\mathrm{AI}[\mathrm{Ec} 1]
$$

Donde:

IA $=$ Indicador de afectación por evento natural

$\mathrm{AD}=$ Indicador de Afectación Directa

$\mathrm{AI}=$ Indicador de Afectación Indirecta

A su vez:

$$
\mathrm{AD}=\mathrm{M}+\mathrm{D}+\mathrm{H}[\mathrm{Ec} 2]
$$


Gustavo Barrantes-Castillo, Adolfo Quesada-Román, Daniela Campos-Durán, Katherine Padilla-Umaña. Indicador de afectación por eventos naturales en el cantón de Alajuela, y su relación con la vulnerabilidad comunal

Donde:

$$
\begin{aligned}
& M=\text { Cantidad de muertos } \\
& D=\text { Cantidad de personas desaparecidos } \\
& H=\text { Cantidad de personas heridos }
\end{aligned}
$$

Y:

$$
\mathrm{AI}=(\mathrm{A}+\mathrm{E}+\mathrm{R}+\mathrm{Vd}+\mathrm{Va}) / 100[\mathrm{Ec} 3]
$$

Donde:

$\mathrm{A}=$ Afectados; se trata de las personas que han sufrido perturbación de sus actividades o medios de sustento, sin que esto representara un evacuación, reubicación, daño o pérdida de sus viviendas.

$\mathrm{E}=$ Evacuados; son personas que han tenido que ser movilizadas a albergues

$\mathrm{R}=$ Reubicados; es la población que dada la peligrosidad de la localidad donde habitan, han sido trasladados a lugares de menor peligrosidad.

$\mathrm{Vd}=$ Viviendas destruidas

$\mathrm{Va}=$ Viviendas afectadas

Los datos sobre personas fallecidas (muertos), desaparecidos o heridos, tienen un mayor peso debido a que representan afectación directa a las personas, por lo que constituyen el indicador de Afectación Directa. Por su parte, las variables: damnificados, afectados, evacuados, reubicados, viviendas destruidas y afectadas, se dividen entre 100 a fin de obtener una tasa, que representa el indicador de Afectación Indirecta. Esto se realiza dada la diferencia de magnitud entre este grupo de variable y el anterior, y que las afectaciones son materiales en el segundo indicador.

El tercer paso, se basó en la agrupación de todos los eventos por distrito. Una vez agrupados se sumaron los indicadores individuales y se dividieron por el número total de eventos, de este modo se construye un indicador distrital relativo que considera las frecuencias de los eventos. Una vez obtenido el indicador distrital, este es clasificado en cinco categorías que se representa por medio de un mapa cantonal. 
Gustavo Barrantes-Castillo, Adolfo Quesada-Román, Daniela Campos-Durán,

Katherine Padilla-Umaña. Affectation indicator for natural events in the cantón of

Alajuela, and its relation with the community vulnerability

La cuarta etapa, consistió en la caracterización de las áreas prioritarias, es decir, de los cinco distritos con mayor valor del indicador. Como insumos de esta etapa se utilizó la ubicación aproximada de los eventos, el valor del indicador de afectación por evento natural y los reportes de inspecciones realizadas por la Comisión Nacional de Prevención de Riesgos y Atención de Emergencias (CNE).

Con base en esta información, se contactaron y se llevaron a cabo entrevistas con los integrantes de las Asociaciones Desarrollo de Integral (ADI), ubicadas en las áreas de mayor afectación histórica. Cuya finalidad fue obtener información sobre las áreas de mayor afectación en la comunidad, así como el conocimiento y preparación de la población sobre la prevención y respuesta en caso de desastre.

En la última etapa, se procedió a elaborar una ficha de campo que permitió la caracterización de la amenaza y la vulnerabilidad a un nivel preliminar con base en aspectos como: la geomorfología, pendiente, tipo de eventos, percepción de la frecuencia, infraestructura afectada y condición socioeconómica, adicionalmente, se documentó por medio de la toma de fotografías y el uso de GPS. Finalmente, se inspeccionaron los sitios para caracterizar las condiciones de riesgo presentes. Las variables valoradas fueron posteriormente clasificadas en rangos de alto, moderado y bajo, para hacer una estimación sobre la amenaza y sobre la vulnerabilidad, ambos criterios fueron combinados para establecer un listado que permitiera ubicar las comunidades de atención prioritaria.

\section{Resultados}

\subsection{Indicador distrital de afectación por incidencia de eventos naturales para cantón de Alajuela}

Los reportes de eventos de la base de datos de DesInventar fueron ubicados de manera aproximada, considerando la ubicación por distrito (y barrio cuando estaba disponible) aportada en la base de datos y el tipo de evento. Posteriormente, se calculó, para cada evento el indicador de afectación por evento (Mapa 1). Estos valores se agruparon en un solo valor para cada distrito, mismo que fue ponderado por el número total de eventos, lo que permitió considerar la frecuencia de los mismos. 
Gustavo Barrantes-Castillo, Adolfo Quesada-Román, Daniela Campos-Durán, Katherine Padilla-Umaña. Indicador de afectación por eventos naturales en el cantón de Alajuela, y su relación con la vulnerabilidad comunal

Mapa 1. Indicador de afectación por eventos naturales en el cantón de Alajuela

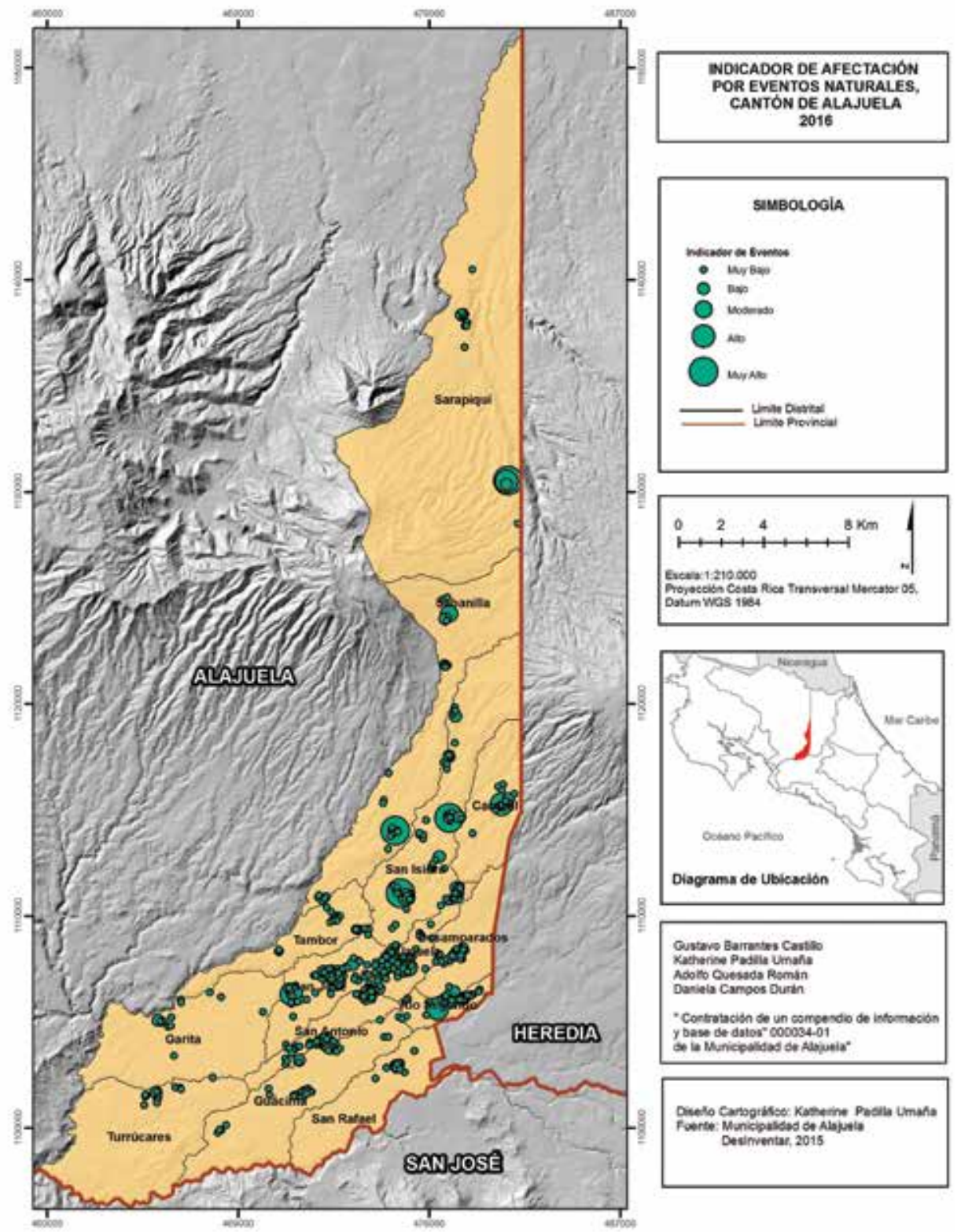

Fuente: Elaboración propia a partir de DesInventar, 2016. 
Gustavo Barrantes-Castillo, Adolfo Quesada-Román, Daniela Campos-Durán,

Katherine Padilla-Umaña. Affectation indicator for natural events in the cantón of

Alajuela, and its relation with the community vulnerability

A cada evento individual le fue calculado el Indicador de afectación por evento natural, de acuerdo con las Ecuaciones [1, 2 y 3], posteriormente, se agruparon todos los eventos por distrito y se dividieron entre el número de eventos para tomar en cuenta la frecuencia de los mismos. El indicador agrupado por distrito se puede apreciar en el Cuadro $3 \mathrm{y}$ en el Mapa 2. Los cinco distritos con mayor incidencia por eventos naturales fueron San Isidro, Alajuela, San José, Sabanilla y San Antonio, que corresponden con las categorías de alto y muy alto en el Mapa 2.

Cuadro 3. Indicador distrital de afectación por amenazas naturales en el cantón de Alajuela, periodo 1970-2015

\begin{tabular}{|l|c|}
\hline \multicolumn{1}{|c|}{ Distrito } & Valor del indicador \\
\hline San Isidro & 3940,6 \\
\hline Alajuela & 3053,1 \\
\hline San José & 2708,1 \\
\hline Sabanilla & 1040,1 \\
\hline San Antonio & 999,7 \\
\hline Sarapiquí & 668,0 \\
\hline Río Segundo & 570,4 \\
\hline Carrizal & 188,0 \\
\hline Guácima & 163,5 \\
\hline San Rafael & 137,7 \\
\hline Desamparados & 100,1 \\
\hline Tambor & 88,1 \\
\hline Turrúcares & 47,5 \\
\hline Garita & 33,4 \\
\hline
\end{tabular}

Fuente: Elaboración propia de los autores, 2016

El indicador de afectación distrital es un valor semi-cuantitativo que permite comparar la afectación acumulada durante todo el periodo de tiempo considerado (1970 - 2015) por distrito, su funcionalidad es ofrecer la posibilidad de clasificar los distritos en cinco rangos de afectación, como se aprecia en el Mapa 2. 
Gustavo Barrantes-Castillo, Adolfo Quesada-Román, Daniela Campos-Durán, Katherine Padilla-Umaña. Indicador de afectación por eventos naturales en el cantón de Alajuela, y su relación con la vulnerabilidad comunal

Mapa 2. Indicador distrital de afectación por amenazas naturales en el cantón de Alajuela

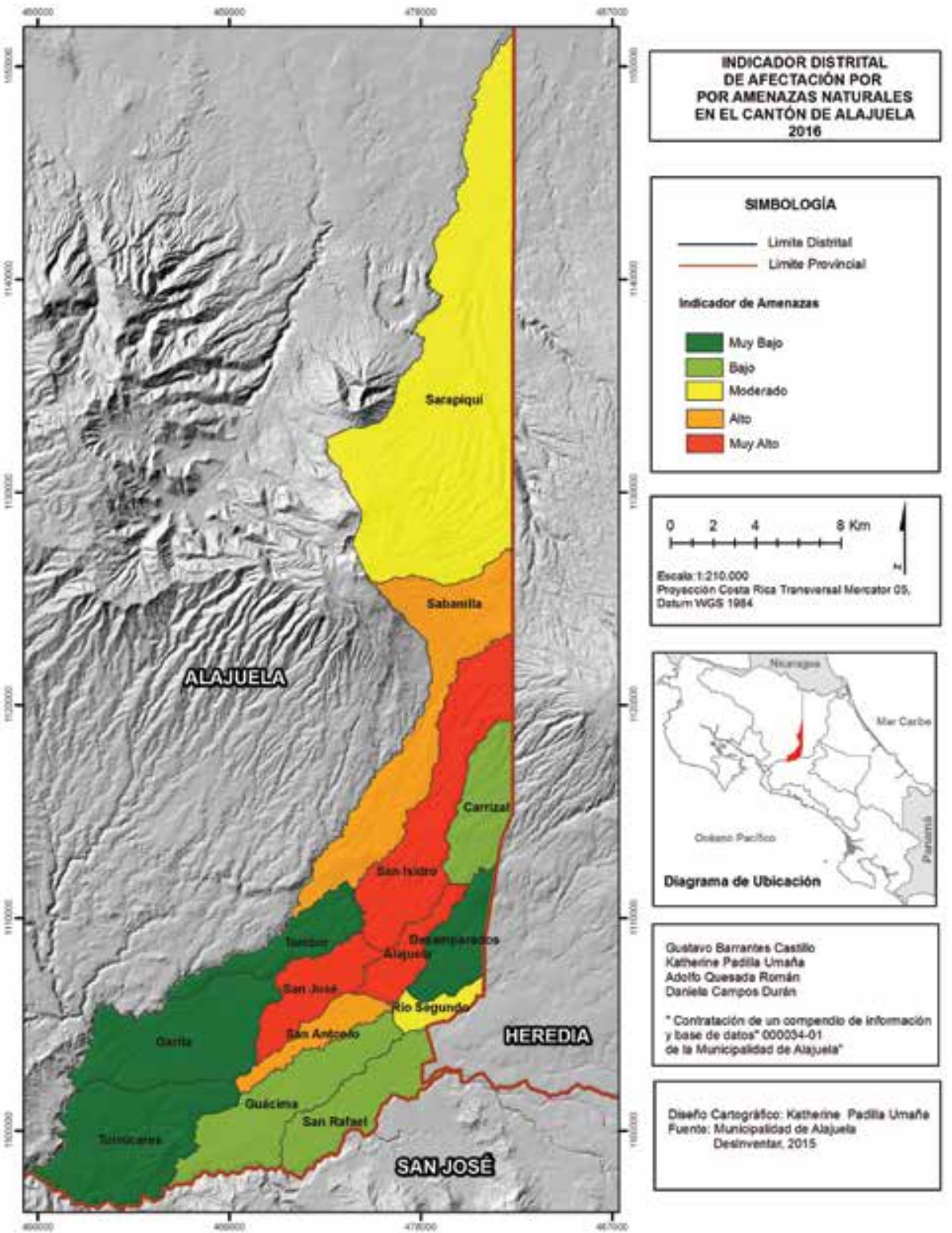

Fuente: Elaboración propia de los autores, 2016 
Gustavo Barrantes-Castillo, Adolfo Quesada-Román, Daniela Campos-Durán,

Katherine Padilla-Umaña. Affectation indicator for natural events in the cantón of

Alajuela, and its relation with the community vulnerability

Con el fin de precisar la información, en términos de localización, se complementaron los datos con los reportes de inspecciones realizados por la CNE en el periodo 2005-2015. Esta información sumada a los datos de DesInventar permitió la planificación de las visitas de campo y la ubicación de las Asociaciones de Desarrollo Integral que serían el punto focal para la obtención de información.

\subsection{Caracterización de amenazas y vulnerabilidad en las co- munidades de mayor afectación por eventos naturales}

Las condiciones de amenaza y vulnerabilidad a nivel local se enfocaron en los cinco distritos que históricamente han presentado mayor afectación por eventos naturales, de acuerdo con el indicador distrital de afectación por eventos naturales, estos distritos fueron-Alajuela, San Antonio, San Isidro, Sabanilla y San José. La ubicación precisa de los sitios fue obtenida en las entrevistas realizadas a los miembros de las ADI y de los informes de inspección de la $\mathrm{CNE}$, en el Cuadro 3 se detallan las asociaciones consultadas.

Cuadro 3. Asociaciones de Desarrollo Integral visitadas en los distritos prioritarios

\begin{tabular}{|l|l|}
\hline \multicolumn{1}{|c|}{ Distrito } & \multicolumn{1}{c|}{ Asociación de Desarrollo Integral consultada } \\
\hline \multirow{4}{*}{ Alajuela } & ADI Alajuela centro \\
\cline { 2 - 2 } & ADI Canoas y Guadalupe \\
\cline { 2 - 2 } & ADI Los Higuerones \\
\cline { 2 - 2 } San Antonio & ADI Montecillos \\
\hline \multirow{4}{*}{ San José } & ADI Ciruelas y Asociación Ambientalista de Ciruelas \\
\cline { 2 - 2 } & ADI Montserrat \\
\cline { 2 - 2 } San Isidro & ADI Villa Bonita \\
\hline \multirow{4}{*}{ Sabanilla } & ADI El Coyol \\
\cline { 2 - 2 } & ADI Barrio San José \\
\hline & ADI San Isidro \\
\cline { 2 - 2 } & ADI San Martín \\
\hline & ADI Poasito \\
\cline { 2 - 2 } & ADI Fraijanes \\
\cline { 2 - 2 } & ADI Sabanilla \\
\cline { 2 - 2 } & ADI San Rafael de Sabanilla \\
\hline
\end{tabular}

Fuente: Daniela Campos Durán, 2016.

172 Revista Geográfica de América Central No 59 ISSN 1011-484X, julio-diciembre 2017, pp. 159-196 
Con base en las entrevistas y las fichas de trabajo de campo, se procedió a realizar una caracterización, sitios con mayor afectación en términos de la amenaza y vulnerabilidad presente. En primera instancia se ubicaron los sitios con mayor afectación por amenazas naturales, según los líderes comunales de las Asociaciones de Desarrollo Integral (ADI) de todos los distritos. Por tanto, se visitaron estos sitios, donde se aplicaron las fichas de trabajo, mediante las cuales se registró la ubicación precisa por medio de la utilización de un navegador (GPS), la caracterización de la geoforma y tipo de pendiente, descripción de la forma de relieve, descripción del sitio y el registro del número o serie de las fotografías tomadas. A continuación, se presenta los resultados por distrito y localidad.

\section{Distrito de Sabanilla}

El distrito Sabanilla está ubicado al norte del cantón de Alajuela, colinda con el Parque Nacional Volcán Poás y por ende con la Cordillera Volcánica Central. Presenta, junto con el distrito de San Isidro, las mayores pendientes del cantón, así como una red de drenajes fluviales densa con valles disecados profundos que en ocasiones entallan en cañones o gargantas fluviales, que incluso en algunos casos alcanza los cien metros de profundidad. En este distrito la mayor cantidad de afectaciones se relacionan con crecidas de ríos y quebradas, así como deslizamientos, estos últimos en su mayoría asociados con el terremoto de Cinchona del año 2009. Por ende, el artículo 2 del Diario oficial la Gaceta menciona que este evento provocó daños en los bienes y las personas en forma directa, debido a la caída de estructuras en la zona más cercana al epicentro, tales como casas e infraestructura colapsada, sobre todo en terrenos quebrados o en laderas, daños en la red vial por derrumbes y obstrucciones, con la perdida inclusive de tramos de carreteras y puentes, así como la ocurrencia de avalanchas en diferentes ríos por deslizamientos, lo cual provocó el aislamiento de las comunidades afectadas, reportándose, incluso, la pérdida de vidas humanas, perdida de las comunicaciones, la agricultura, los servicios públicos, por tal motivo, la Comisión Nacional de Prevención de Riesgos y Atención de Emergencias declaró en forma inmediata después de producirse el evento, alerta roja para los cantones con impacto directo por el sismo (Artículo 2 del Decreto de Emergencia No. 34993.CNE, 2009) 
Gustavo Barrantes-Castillo, Adolfo Quesada-Román, Daniela Campos-Durán,

Katherine Padilla-Umaña. Affectation indicator for natural events in the cantón of

Alajuela, and its relation with the community vulnerability

Las comunidades y los sitios críticos visitados en este distrito se presentan en el Cuadro 4.

Cuadro 4. Comunidades y sitios visitados

\begin{tabular}{|l|l|}
\hline \multicolumn{1}{|c|}{ Comunidad visitada } & \multicolumn{1}{c|}{ Sitios críticos inspeccionados } \\
\hline \multirow{2}{*}{ Poasito } & Quebrada Las Minas \\
\cline { 2 - 2 } & Quebrada El Sabor de la Montaña \\
\hline Fraijanes & Calle Montenegro \\
\hline Sabanilla & Calle Tambor \\
\hline \multirow{3}{*}{ San Rafael de Sabanilla } & Acequia frente a iglesia católica \\
\cline { 2 - 2 } & Calle El Cerro- Calle Vargas \\
\cline { 2 - 2 } & Calle Potrerillos \\
\hline
\end{tabular}

Fuente: Daniela Campos Durán, a partir de Asociaciones de Desarrollo Integral de Poasito, Fraijanes, Sabanilla y San Rafael de Sabanilla, 2016.

La comunidad de Poasito, tal como se señaló anteriormente, fue una de las comunidades más afectadas por el terremoto de Cinchona en el año 2009, para ello Barrantes et al, (2013), señalan que parte de las afectaciones que se dieron en la población y el ambiente fueron por deslizamientos de varios tipos y tamaños.

Asimismo, esta comunidad ha sido impactada por otros eventos asociados a dos cauces fluviales correspondientes a Quebrada Minas y la Quebrada ubicada al norte del Restaurante El Sabor de la Montaña, en las cuales se presentaron cabezas de agua (inundaciones rápidas). Para el caso de Quebrada Minas, los eventos se dieron en los años 1998 y 2007 y en la Quebrada al norte del Restaurante El Sabor de la Montaña en el 2009 (luego del terremoto de Cinchona) y en el 2013. En este último punto se encuentra un cauce fluvial entubado, lo cual provoca inundaciones en periodos de lluvia extraordinaria, dada la incapacidad de las tuberías de drenar toda el agua que baja por estos canales.

En el caso de Quebrada Minas, socava varios muros de contención de propiedades privadas sobre la carretera que se dirige hacia el volcán Poás, las crecidas de este afluente se han visto en aumento debido a la modificación de su cauce por parte de la entubación y al aporte de sedimentos por los cambios en el uso de la tierra de fincas ganaderas aguas arriba, lo cual ha transformado su dinámica. 
Gustavo Barrantes-Castillo, Adolfo Quesada-Román, Daniela Campos-Durán, Katherine Padilla-Umaña. Indicador de afectación por eventos naturales en el cantón de Alajuela, y su relación con la vulnerabilidad comunal

Otro punto crítico en el distrito de Sabanilla se localiza en la comunidad de Fraijanes, específicamente, la Calle Montenegro, la cual está ubicada en un cauce fluvial intermitente, de unos 6 metros de ancho y una profundidad de aproximadamente 8 metros, tal como se muestra en la Figura 3 , el cual es activado durante la época lluviosa. Tanto vecinos como la misma municipalidad han construido desagües a ambos lados de la calle con profundidades variables entre 1 y $5 \mathrm{~m}$, con anchos de hasta $2 \mathrm{~m}$ a fin de conducir las aguas durante las lluvias.

Figura 3. Cauce fluvial intermitente en Calle Montenegro, Fraijanes

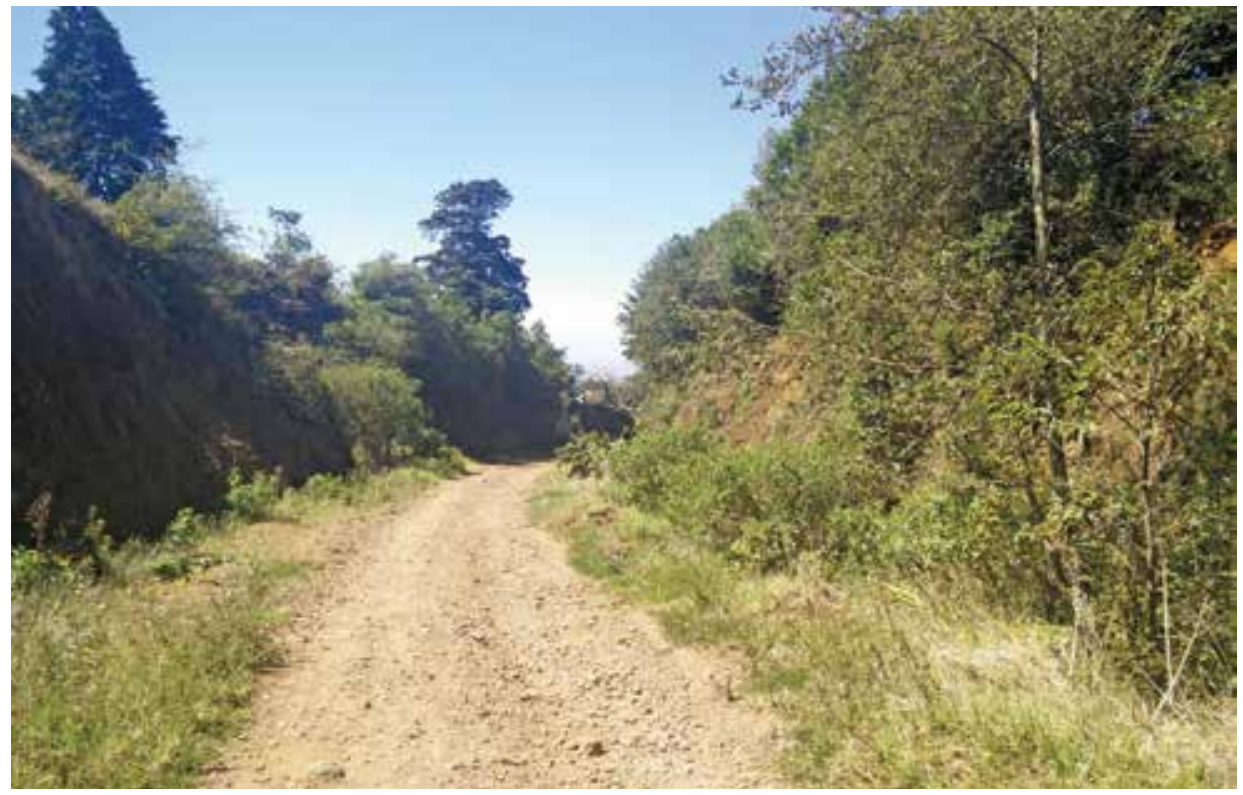

Fuente: Adolfo Quesada Román, 15 de marzo de 2016.

Ambos cauces fueron entubados unos 400 metros antes de que desfoguen en el alcantarillado de la carretera principal, lo que ha provocado pequeños procesos de ladera en el margen derecho, sector donde se entuba el desagüe. Además, en la salida de esta alcantarilla se realizó un muro para la salida de las aguas drenadas, donde se muestran clastos de más de un metro de diámetro, lo que indica la capacidad de carga de este torrente, muy anguloso que muestra que ha recibido poco transporte. 
Gustavo Barrantes-Castillo, Adolfo Quesada-Román, Daniela Campos-Durán,

Katherine Padilla-Umaña. Affectation indicator for natural events in the cantón of

Alajuela, and its relation with the community vulnerability

Figura 4. Unión de cauces en calle Montenegro, Fraijanes.

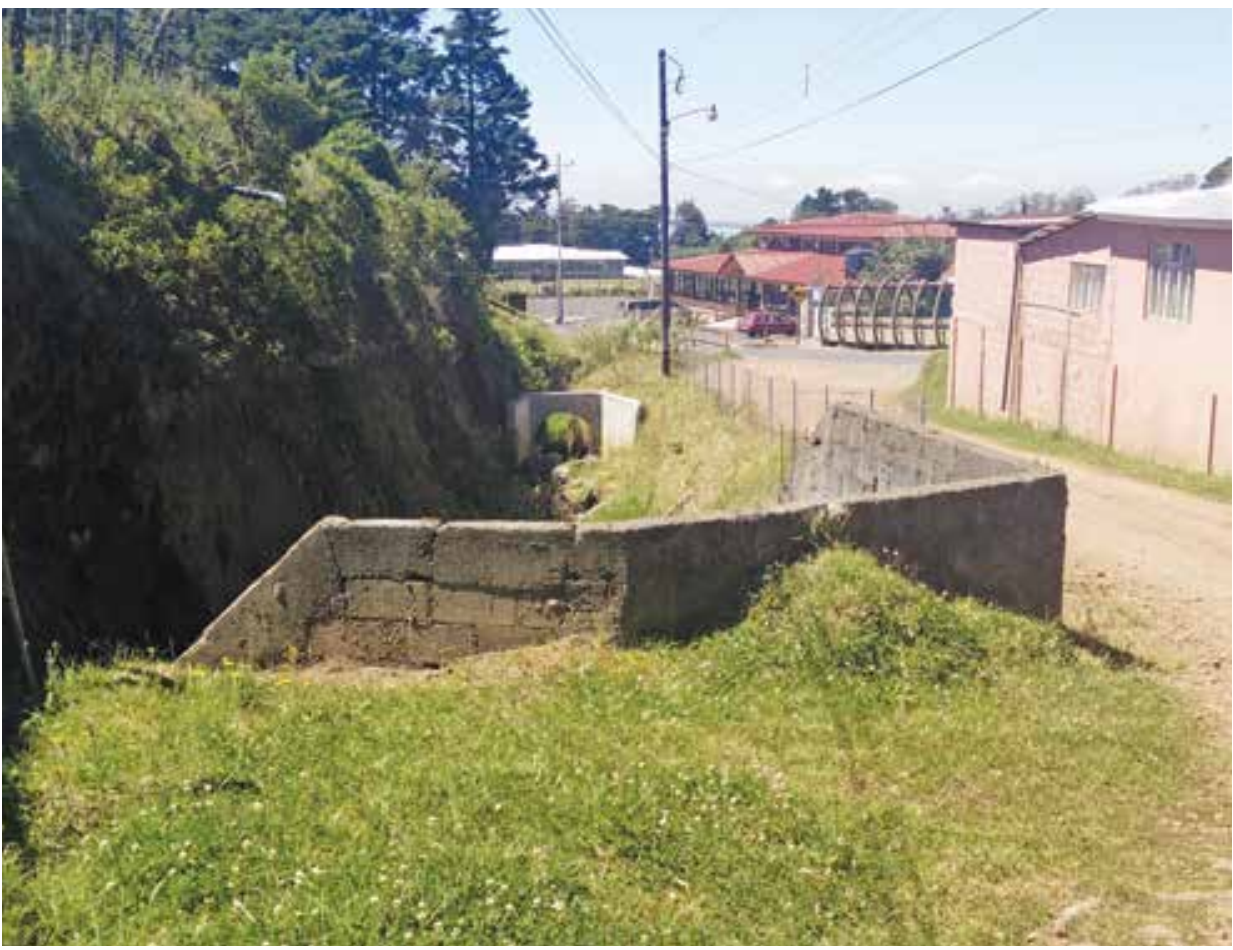

Fuente: Adolfo Quesada Román, 15 de marzo de 2016.

Otros sitios críticos se localizaron en la comunidad de Sabanilla, en la cual se han presentado eventos por inundaciones y deslizamientos. Las inundaciones asociadas al deficiente alcantarillado se dan todos los años en Calle Tambor, las cuales se han intensificado debido al proceso de urbanización en la parte alta de la comunidad de Sabanilla y por la cantidad de basura que se acumula en las alcantarillas.

En la comunidad de San Rafael de Sabanilla también se han presentado eventos asociados a inundaciones, provocadas por el desbordamiento de la acequia que pasa frente a la iglesia católica, la cual recoge aguas de la zona alta del distrito. El último desbordamiento de la acequia se reporta en el 2015, entre los meses de mayo y junio, lo cual afectó cuatro viviendas cercanas. La municipalidad gestionó labores para el entubamiento de la acequia, sin embargo, ello provocó malestar en los vecinos, por lo que la obra no se llevó a cabo. 
Otro punto crítico en esta comunidad que se ha visto afectado por deslizamientos es la carretera que comunica El Cerro con el distrito de Tambor, esta vía está trazada sobre la divisoria de aguas y al no existir un sistema de alcantarillado adecuado, las aguas servidas y de escorrentía se filtran y generan inestabilidad en las laderas, lo cual es un factor que se ve favorecido debido a la topografía de altas pendientes. Cabe señalar, que esta carretera se reporta inestable desde el año 2012, la municipalidad colocó obras de gaviones en algunos puntos que se han deslizado.

Se identificó además otra área en condiciones de riesgo, correspondiente a la Calle Potrerillos, la cual va desde la iglesia hasta el tajo. Esta calle presenta una marcada pendiente superior a los $45^{\circ}$, por la cual bajan las aguas de escorrentía en época lluviosa, además de ello las aguas residuales y negras son depositadas en un caño al aire libre, lo cual genera malos olores y brotes de plagas, tal como zancudos y roedores. Las viviendas construidas en esta calle, especialmente las últimas casas, presentan un riesgo a deslizamiento por su cercanía con el tajo, el cual tiene cerca de 200 metros de profundidad.

\section{Distrito San Isidro}

El distrito de San Isidro está localizado al centro-norte del cantón de Alajuela. Desde el punto de visto geodinámico, combina pendientes altas en la sección norte del distrito con pendientes bajas al sur, suelos derivados de piroclastos provenientes de los volcanes Poás y Barva, así como marcados valles fluviales que entallan cauces con profundidades que varían desde pocos hasta más de cien metros. La mayor amenaza está representada por los deslizamientos en comunidades como: Laguna, Dulce Nombre, Pilas y San Martín, no obstante, la problemática con las zonas de inundación es ampliamente extendida en la parte baja del distrito correspondiente a la comunidad de San Martín. Los sitios críticos que se identificaron se presentan en el Cuadro 5. 
Gustavo Barrantes-Castillo, Adolfo Quesada-Román, Daniela Campos-Durán,

Katherine Padilla-Umaña. Affectation indicator for natural events in the cantón of

Alajuela, and its relation with the community vulnerability

Cuadro 5. Comunidades visitadas y sitios críticos

\begin{tabular}{|l|l|}
\hline \multicolumn{1}{|c|}{ Comunidad visitada } & \multicolumn{1}{c|}{ Sitios críticos o históricamente afectados } \\
\hline \multirow{5}{*}{ San Martín } & Puente Alajuela \\
\cline { 2 - 2 } & Calle Ceiba \\
\cline { 2 - 2 } & Departamentos frente a Auto Mercado \\
\cline { 2 - 2 } & Puente de la tenería \\
\hline \multirow{5}{*}{ San Isidro } & Desde El Mirador hasta Calle Bambú \\
\cline { 2 - 2 } & Hotel La Lomita \\
\hline & Mirador El Condor \\
\cline { 2 - 2 } & Carretera principal \\
\cline { 2 - 2 } & Laguna - Dulce Nombre \\
\cline { 2 - 2 } & Asentamiento informal - costado río Tambor \\
\hline
\end{tabular}

Fuente: Daniela Campos Durán, a partir de Asociaciones de Desarrollo Integral de San Isidro y San Martin, 2016.

En la comunidad de San Isidro, el principal evento que ha impactado fue el terremoto de Cinchona del 2009, el cual disparó deslizamientos, principalmente en la comunidad de Laguna, no obstante, históricamente San Isidro se ha visto afectado por deslizamientos, principalmente en época lluviosa. Se constató mediante trabajo de campo que la ladera SE de la carretera que comunica a las comunidades de Laguna y Dulce Nombre presenta procesos de ladera, los cuales se activaron, probablemente, por el Terremoto de Cinchona en el 2009. Cabe resaltar, que todo este sector se compone de suelos andisoles, muy friables e inestables como taludes para sostener construcciones.

Otros sitios críticos con presencia de deslizamientos, asociados con la época lluviosa, se ubican en la comunidad de Pilas, específicamente en El Mirador y calle El Bambú, en Hotel Las Lomitas y algunos tramos a lo largo de la carretera que comunica San Isidro con el distrito de Alajuela, en esta vía la municipalidad y el MOPT (Ministerio de Obras Públicas y Transportes) han colocado, en al menos tres puntos, gaviones para detener los hundimientos en carretera. Pilas se localiza en pendientes francas de más de $45^{\circ}$, el corte de la ladera con la construcción de la carretera provoca desprendimientos por la pérdida del ángulo de reposo de los suelos volcánicos meteorizados, tal como se muestra en la Figura 5. Es importante señalar que su reactivación está asociada con lluvias intensas y procesos sísmicos con intensidades mayores a V IMM.

Revista Geográfica de América Central No 59 ISSN 1011-484X, julio-diciembre 2017, pp. 159-196 
Gustavo Barrantes-Castillo, Adolfo Quesada-Román, Daniela Campos-Durán, Katherine Padilla-Umaña. Indicador de afectación por eventos naturales en el cantón de Alajuela, y su relación con la vulnerabilidad comunal

Figura 5. Terrenos inestables y de marcada pendiente en la comunidad Pilas, distrito San Isidro

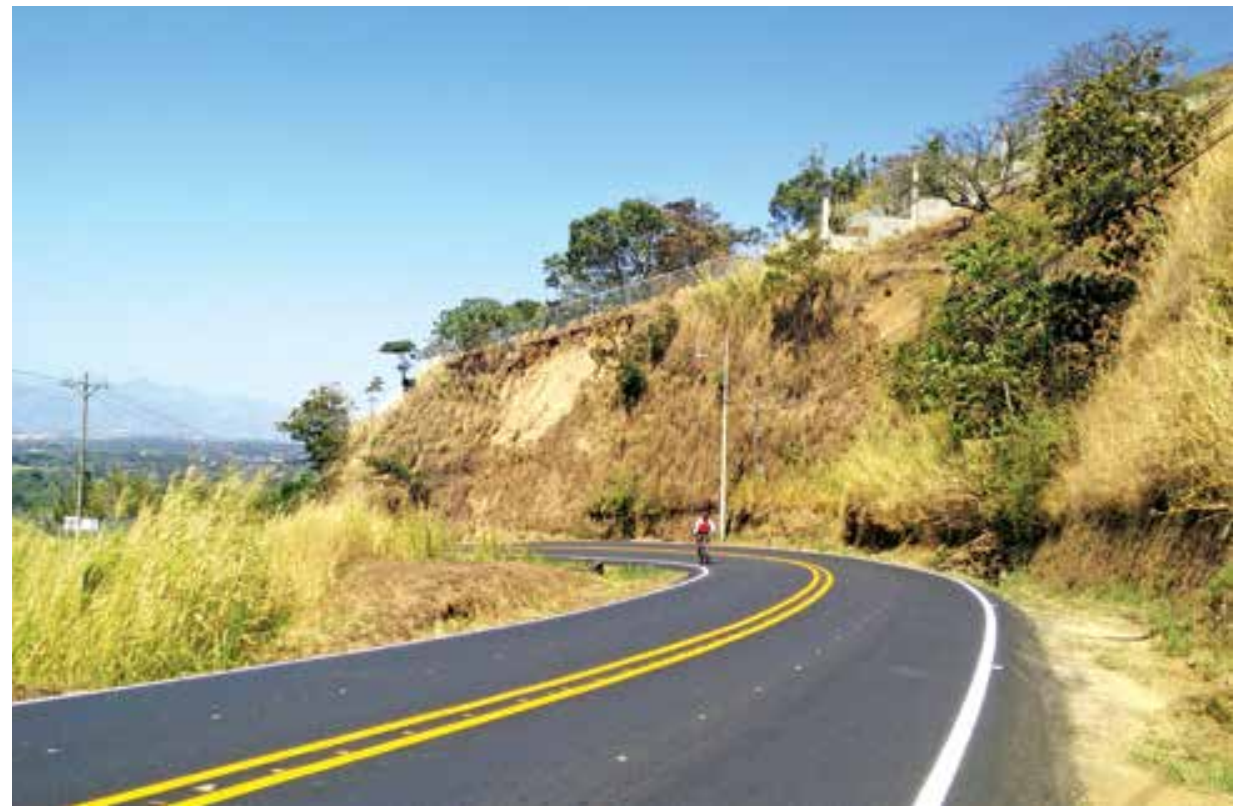

Fuente: Adolfo Quesada Román, 8 de abril de 2016.

Otros eventos que se han presentado en este distrito son las inundaciones, específicamente en la parte baja, en la comunidad de San Martin, los sitios críticos en este sector se ubican en los alrededores de la carretera principal desde Calle La Ceiba hasta el puente Alajuela, donde es frecuente que en época lluviosa se inunde por el colapso de la red de alcantarillado, ya que esta recoge las aguas de la parte alta del distrito. Cabe señalar, que la última inundación sobre el puente Alajuela se dio en el mes de octubre del2015.

Algunos riesgos asociados que se identificaron en este distrito fueron a la altura de Espino, en la parte alta del distrito, a poco más de un kilómetro de la comunidad de Dulce Nombre, donde se encuentra el puente sobre el río Tambor, mismo que muestra socavación de sus bases. Especial atención requiere el caserío informal localizado sobre un cauce fluvial intermitente, el cual en periodos de lluvias extraordinarias podría generar inundaciones súbitas, deslizamientos y flujos de lodo. Otro punto crítico asociado a riesgo por inundación es el sector de puente la Tenería en la 
Gustavo Barrantes-Castillo, Adolfo Quesada-Román, Daniela Campos-Durán,

Katherine Padilla-Umaña. Affectation indicator for natural events in the cantón of

Alajuela, and its relation with the community vulnerability

comunidad de San Martín, es una estructura vieja que podría colapsar ante una crecida del rio, afectando las viviendas que colindan con el río. Se identificó además un muro inestable en unos departamentos frente al Auto Mercado, el cual está en riesgo de colapsar.

\section{Distrito San José}

El distrito San José se localiza al centro-sur del cantón de Alajuela, sobre depósitos aluviales del Plioceno-Cuaternario en relieves semiplanos con inclinaciones menores a $20^{\circ}$, alternando con valles fluviales con pendientes de hasta $60^{\circ}$. Las inundaciones se reportan como recurrentes, principalmente en las comunidades de El Coyol y Barrio San José, los puntos críticos que se identificaron en este distrito se encuentran en el Cuadro 6.

Cuadro 6. Comunidades visitadas y sitios críticos

\begin{tabular}{|l|l|}
\hline \multicolumn{1}{|c|}{ Comunidad visitada } & \multicolumn{1}{c|}{ Sitios críticos o históricamente afectados } \\
\hline \multirow{5}{*}{ Barrio San José } & Calle Fraccionamiento \\
\cline { 2 - 2 } & Calle principal desde La Princesa Marina a la gasolinera \\
\cline { 2 - 2 } & Puente Mega Súper \\
\cline { 2 - 2 } & Puente Urbanización Meza \\
\hline \multirow{5}{*}{ El Coyol } & Carretera principal desde El Pacto del Jocote \\
\cline { 2 - 2 } & Urbanizaciones: Sierra Morena, Bertilia, Villa Rica, \\
\cline { 2 - 2 } & Calle Murillo \\
\cline { 2 - 2 } & Calle Santa Cecilia \\
\cline { 2 - 2 } & Puente sobre Calle El Salto \\
\cline { 2 - 2 } & Puente La Mandarina \\
\hline
\end{tabular}

Fuente: Daniela Campos Durán, a partir de Asociaciones de Desarrollo Integral de Coyol y Barrio San José, 2016.

Los eventos que se presentan, principalmente, en época lluviosa, son las inundaciones, las cuales han afectado de manera recurrente sitios específicos en el distrito de San José, tal como Calle Fraccionamiento, que se inunda frecuentemente, solo en el 2015 se reportan 15 inundaciones en este sector, donde el agua ha alcanzado alrededor de un metro de altura. Las inundaciones en este caso se relacionan con el canal de riego que atraviesa esta calle, cuya capacidad es sobrepasada en la parte más lluviosa del año, lo mismo sucede con el alcantarillado pluvial durante lluvias intensas. 
Gustavo Barrantes-Castillo, Adolfo Quesada-Román, Daniela Campos-Durán, Katherine Padilla-Umaña. Indicador de afectación por eventos naturales en el cantón de Alajuela, y su relación con la vulnerabilidad comunal

Lo mismo sucede con la carretera principal de Barrio San José (Cuadro 6), la cual comunica con otros cantones tal como Poás, Grecia y Atenas, que se inunda como resultado del colapso del sistema de alcantarillado durante aguaceros intensos, a consecuencia de recibir las aguas pluviales de las partes altas del cantón. Este proceso se ha visto agravado debido al aumento del área urbana que reduce la capacidad de infiltración de los suelos. Un aspecto a considerar en esta comunidad son los cuatro puentes ubicados sobre el río Alajuela, donde el proyecto "Pluviales del Este" depositaría parte de las aguas de distrito de Alajuela en este cauce, el aumento en el caudal podría socavar los puentes que no reciben mantenimiento. Estos puentes son el que se ubica frente al Mega Súper, puente de La Arrocera, puente en urbanización Meza y puente El Salto.

Para el caso de la comunidad de El Coyol, se determinó que, unos $3.5 \mathrm{~km}$ de distancia a lo largo de la carretera que comunica esta localidad con el centro de Alajuela presentan inundaciones producto de la presencia de una zona semiplana con pendientes menores a $10^{\circ}$, que durante precipitaciones fuertes es común que los canales a ambos lados de dicha calle tienden a desbordarse sobre la carretera. Dichas inundaciones afectan las viviendas y urbanizaciones que se encuentran a orilla de la carretera, tales como Villa Rica Norte y Sur, La Guaria, Los Olivos, entre otras. A esta situación se le debe sumar la cantidad de basura que se deposita en estos desagües, así como la contaminación asociada al mal tratamiento de aguas negras y servidas, lo cual provoca contaminación (Figura 6). 
Gustavo Barrantes-Castillo, Adolfo Quesada-Román, Daniela Campos-Durán,

Katherine Padilla-Umaña. Affectation indicator for natural events in the cantón of

Alajuela, and its relation with the community vulnerability

Figura 6. Limpieza de canal frente a la calle principal, El Coyol, distrito San José

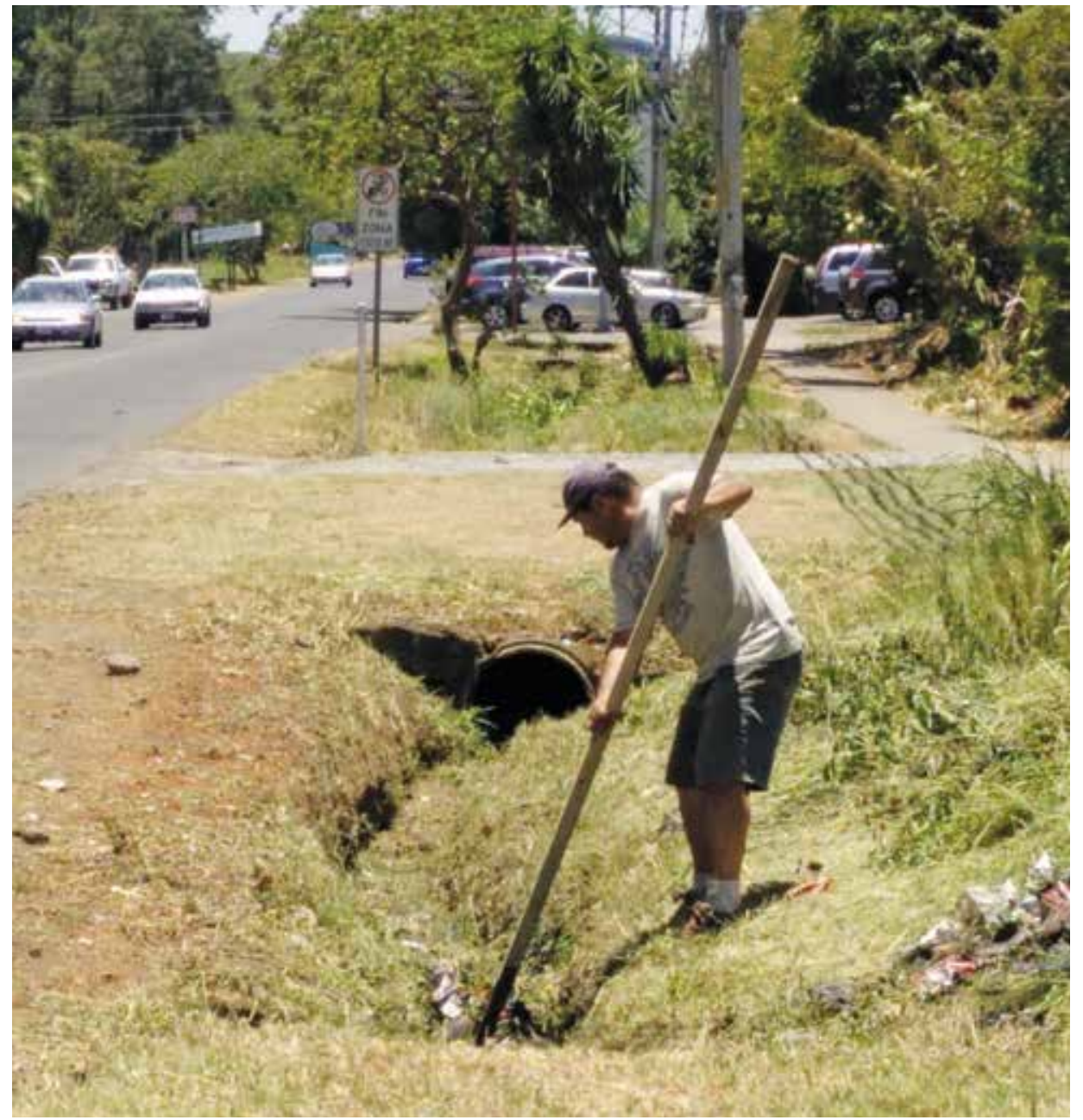

Fuente: Adolfo Quesada Román, 9 de abril de 2016.

Sumado al anterior proceso, está el desbordamiento del río Alajuela, el cual inunda las viviendas de las urbanizaciones que colindan con este cauce. Otro sitio crítico, en Barrio San José, es el puente La Mandarina, cuya estructura es vieja y se ve rebalsada por el agua durante periodos de lluvia fuerte. Asimismo, durante una avenida extraordinaria los asentamientos que colindan con el río se podrían ver afectados. 
Gustavo Barrantes-Castillo, Adolfo Quesada-Román, Daniela Campos-Durán, Katherine Padilla-Umaña. Indicador de afectación por eventos naturales en el cantón de Alajuela, y su relación con la vulnerabilidad comunal

\section{Distrito Alajuela}

El distrito de Alajuela fue el que presentó la mayor afectación por inundación, así como afectaciones importantes por deslizamientos. Las comunidades y los sitios críticos que se identificaron se resumen en el Cuadro 7.

Cuadro 7. Comunidades visitadas y sitios críticos

\begin{tabular}{|l|l|}
\hline \multicolumn{1}{|c|}{ Comunidad visitada } & \multicolumn{1}{c|}{ Sitios críticos o históricamente afectados } \\
\hline \multirow{4}{*}{ Alajuela centro } & Calle Real, Avenida 5 y Avenida 3 \\
\cline { 2 - 2 } & Barrio San Luis - El Estero \\
\cline { 2 - 2 } & Puente a la altura de La Arrocera \\
\cline { 2 - 2 } & El Arroyo \\
\hline \multirow{4}{*}{ Gontecillos } & Puente sobre Quebrada Barro \\
\cline { 2 - 2 } & Residencial Baviera \\
\hline \multirow{4}{*}{ Los Higuerones } & Urbanización Monte Cristal \\
\cline { 2 - 2 } & Puente Negro \\
\cline { 2 - 2 } & Urbanización Los Ángeles \\
\hline & Carretera frente a la Clínica Marcial Rodríguez \\
\cline { 2 - 2 } & Desde licorera El Bambú a Lotes Guardia y Estadio \\
\cline { 2 - 2 } & Urbanización Lirios del Valle \\
\hline
\end{tabular}

Fuente: Daniela Campos Durán, a partir de Asociaciones de Desarrollo Integral de Alajuela Centro, Canoas y Guadalupe, Los Higuerones y Montecillos, 2016.

En el distrito de Alajuela se consultaron cuatro Asociaciones de Desarrollo, de las cuales se obtuvieron once sitios críticos que han sido afectados principalmente por inundaciones o deslizamientos, así como por inundaciones urbanas ligadas a la crecida de los ríos y a la incapacidad del alcantarillado público.

El centro de Alajuela se ha visto afectado con regularidad por inundaciones, principalmente, en Calle Real, Avenida 3 (Figura 7) y Avenida 5; los sitios más críticos son el Parque Central (Farmacia Chavarría), el Mercado Municipal y comercios que se ven afectados por el incapacidad de los sistemas de alcantarillado pluvial durante aguaceros de alta intensidad que se presentan por lo general en los meses de setiembre a noviembre, cabe señalar que este problema se ve agravado por los desechos sólidos acumulados en el sistema de drenaje artificial de estos sectores. 
Gustavo Barrantes-Castillo, Adolfo Quesada-Román, Daniela Campos-Durán,

Katherine Padilla-Umaña. Affectation indicator for natural events in the cantón of

Alajuela, and its relation with the community vulnerability

Figura 7. Avenida 3 Alajuela centro

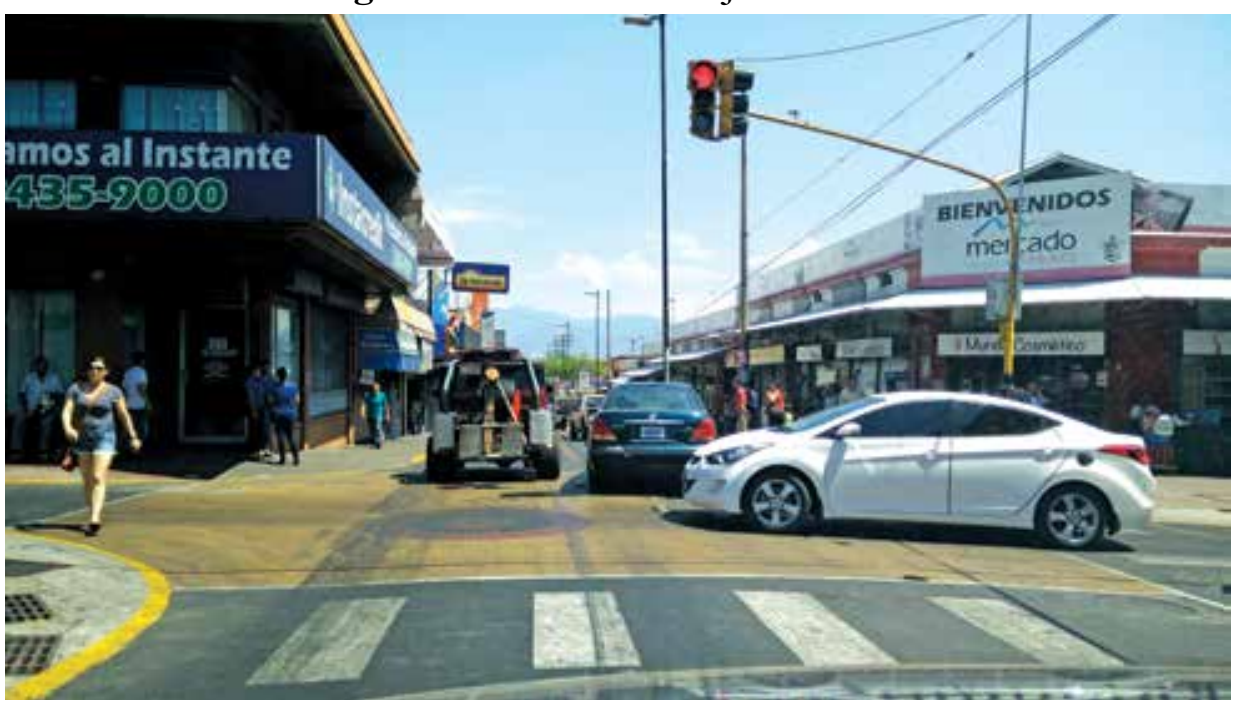

Fuente: Adolfo Quesada Román, 9 de abril de 2016.

Otro sector con problemas recurrentes de inundaciones urbanas es la Urbanización Baviera en Montecillos, asociado también con la inadecuada gestión del sistema de alcantarillados durante eventos extremos, así como con la obstrucción por basura. A consecuencia de este proceso, se identifica un socavamiento de terreno que ha generado daños en la red vial. En el 2013 la Municipalidad de Alajuela mejoró el sistema de alcantarillado y el problema se resolvió, no obstante, este tipo de situaciones son comunes en el cantón y debería ser una línea clara de mejoramiento de la infraestructura municipal.

Otro punto crítico en el distrito de Alajuela por inundaciones es la comunidad de Los Higuerones, específicamente, en la carretera frente a la Clínica Marcial Rodríguez y los sectores desde la licorera El Bambú a Lotes Guardia y el Estadio Alejandro Morera Soto. Las inundaciones en este sector se han incrementado a consecuencia del proceso de urbanización en las partes altas del distrito, que contribuye a la impermeabilización del suelo y aumenta el agua de escorrentía, lo anterior sumado a las aguas residuales, pluviales y los desechos sólidos que colapsan la capacidad del sistema de alcantarillado durante lluvias intensas. El desbordamiento de las aguas afecta la vía pública, aceras e incluso viviendas y comercios aledaños. 
Gustavo Barrantes-Castillo, Adolfo Quesada-Román, Daniela Campos-Durán, Katherine Padilla-Umaña. Indicador de afectación por eventos naturales en el cantón de Alajuela, y su relación con la vulnerabilidad comunal

Así mismo, el desbordamiento de los ríos Alajuela y Ciruelas provoca problemas de inundaciones, el primero se desborda a la altura de La Arrocera y el segundo en los Barrios San Luis y El Estero, contiguo al Plantel de Tuasa. En el sector de la Arrocera, sobre el puente del río Alajuela, se presentan problemas de socavamiento de los márgenes del río, que incluso muestra daños en las obras de estabilización de laderas construidas para proteger un segmento de calle que pasa a un costado del río, por ejemplo, los gaviones ubicados en la margen izquierda del puente. El avance de este proceso podría afectar varios comercios que tienen sus muros de contención limitando con el cauce fluvial, así como el puente mismo, el cual es de alto tránsito.

Este está ubicado en el sector de La Arrocera, y todos los años se ve afectado por el desbordamiento del rio, que no solo afecta a comercios contiguos, sino también al tránsito vehicular, por lo cual se han colocado obras de mitigación en ambos márgenes del río. Para el caso del río Ciruelas, a la altura de los barrios San Luis y el Estero, las viviendas se localizan en ambos márgenes del río, incluso confinan el cauce, lo cual representa un peligro ante un escenario de inundación mayor que podría socavar la base de algunos de los muros de las viviendas. Señala, además, que existen otras estructuras expuestas a daños, tal como el puente que comunica Alajuela con el INVU Las Cañas con un alto tránsito vehicular, y próximamente, el paso del tren.

El distrito también se ha visto afectado por deslizamientos, específicamente en Guadalupe, en el sector de Puente Negro, donde hace siete años se activó un deslizamiento por las lluvias, el mismo destruyó una vivienda y provocó la muerte de una persona, actualmente en el sitio existen tres viviendas expuestas, las cuales se han visto afectadas en menor medida por eventos similares. En Los Higuerones, específicamente en la Urbanización Lirios del Valle, hace cuatro años ocurrió un colapso de una vivienda, debido a fugas en la alcantarilla que pasaba debajo de la misma; el terreno deslizado es un área de relleno.

Otro de los puntos críticos del distrito de Alajuela está asociado con la desestabilización del terreno que se encuentra en la comunidad de Canoas, específicamente, en la Urbanización Los Ángeles, donde un muro de contención está desestabilizado y podría afectar una propiedad privada, la acera y la carretera. En Urbanización Monte Cristal un muro de contención 
Gustavo Barrantes-Castillo, Adolfo Quesada-Román, Daniela Campos-Durán,

Katherine Padilla-Umaña. Affectation indicator for natural events in the cantón of

Alajuela, and its relation with the community vulnerability

de cemento de unos seis metros de longitud está a punto de caer, lo que podría afectar cerca de tres casas de habitación que están siendo soportadas por este muro y otro construido con llantas. En este caso también las viviendas fueron construidas sobre un relleno.

En la comunidad de Guadalupe se encuentra, además, el Puente Negro, estructura inestable que está apoyada en un árbol, se trata de un puente bayley antiguo, que da acceso a fincas agrícolas y por el cual transitan vehículos de carga, lo cual podría generar una emergencia en caso que el puente colapse. Otras condiciones de riesgo que se identificaron son las construcciones existentes en las márgenes de los ríos, como el caso de Montecillos donde existen viviendas y comercios al margen de la Quebrada Barro, adicionalmente el río presenta contaminación visible. Finalmente, en la comunidad de Montecillos, se ubica un puente sobre la Quebrada Barro en la Calle Perica. Esta estructura fue construida hace cuatro años, sin embargo, ya presenta socavamiento en sus bases y se evidencia pérdida de un sector de la carretera por erosión lateral de la quebrada.

\section{Distrito San Antonio}

En el distrito de San Antonio la mayor cantidad de eventos que han presentado afectación corresponden a inundaciones, los sitios críticos que se identificaron fueron los reportados en el Cuadro 8.

Cuadro 8. Comunidades visitadas y sitios críticos

\begin{tabular}{|l|l|}
\hline \multicolumn{1}{|c|}{ Comunidad visitada } & \multicolumn{1}{c|}{ Sitios críticos o históricamente afectados } \\
\hline \multirow{4}{*}{ Montserrat } & Sobre puente peatonal Molinos de Costa Rica \\
\cline { 2 - 2 } & Puente a la altura de Casa Proveedora Phillips \\
\cline { 2 - 2 } & Puente La Arena \\
\cline { 2 - 2 } & Quebrada Los Molinos \\
\hline \multirow{4}{*}{ Villa Bonita } & Villa Bonita Centro \\
\cline { 2 - 2 } & Acequia Grande \\
\cline { 2 - 2 } Ciruelas & Carretera principal: desde UTN a Calle Ancha \\
\hline \multirow{2}{*}{} & Desbordamiento de márgenes del río Siquiares \\
\cline { 2 - 2 } & Industria: Amenaza tecnológica \\
\cline { 2 - 2 } & Residencial Andalucía \\
\hline
\end{tabular}

Fuente: Daniela Campos Durán, a partir de Asociaciones de Desarrollo Integral de Monserrat, Villa Bonita y Ciruelas y Asociación Ambientalista de Ciruelas, 2016. 
En el distrito de San Antonio las amenazas naturales y eventos de desastres que han ocurrido están asociados principalmente con inundaciones, ello debido a sus características geomorfológicas, condiciones pluviométricas y la dinámica urbana que se ha desarrollado en la parte baja del cantón. Las comunidades más afectadas por los eventos de inundación son Ciruelas, Montserrat y Villa Bonita. En la comunidad de Montserrat se identificaron cuatro sitios críticos todos con afectación por inundaciones asociados al desbordamiento del río Ciruelas y el ineficiente sistema de alcantarillado, principalmente en época lluviosa con periodos de intensos aguaceros.

A la altura del puente La Arena, por el cual pasa el río Ciruelas, el último desbordamiento se dio en el año 2014. Los procesos de anegación en este punto están asociados a la estrechez del puente en relación con el caudal durante la época lluviosa y el aporte de aguas residuales e incluso negras que se depositan en este cauce. Cabe señalar que este cause fue dragado por la municipalidad en el año 2014, obra que no recibe mantenimiento, lo que reduce su efectividad para disminuir el impacto de las inundaciones en este punto.

El puente en las proximidades de Casa Proveedora Phillips es otro punto crítico, donde se presentan desbordamientos del río Ciruelas, el último evento se presentó en el año 2015 debido a fuertes lluvias, aunado a la cantidad de basura que arrastra el río. Cabe señalar que históricamente este punto se ha visto afectado por importantes inundaciones como lo fue en los años 1990, 1995 y 2006. Actualmente, se está construyendo un nuevo paso vehicular.

Otros puntos que han presentado afectación por inundación son el puente peatonal Molinos de Costa Rica y quebrada Los Molinos, este último presentó un importante evento de inundación en la comunidad de Montserrat en el mes de octubre del año 2015, parece estar relacionado con la construcción del "City Mall", donde se encausó la quebrada con tuberías que desfoga en el río Ciruelas a la altura de Los Molinos. Es importante señalar que la obra "Cortes Pluviales del Este" aporta parte del caudal de aguas de lluvia, así como pluviales y residuales que recoge de las partes altas del cantón de Alajuela al río Ciruelas, lo que representaría un aumento en el caudal de dicho río y un posible incremento en la frecuencia y magnitud de las inundaciones en sitios como Montserrat, Ciruelas y Villa Bonita. 
Gustavo Barrantes-Castillo, Adolfo Quesada-Román, Daniela Campos-Durán,

Katherine Padilla-Umaña. Affectation indicator for natural events in the cantón of

Alajuela, and its relation with the community vulnerability

Por su parte la comunidad de Villa Bonita, afectada también por inundaciones presenta tres puntos críticos, uno de ellos es la quebrada Grande, cuyo último desbordamiento fue en el mes de octubre del año 2015, sin embargo, está quebrada fue entubada y desviada al río Ciruelas, ello debido a la construcción del "City Mall”. No obstante, está quebrada recoge las aguas residuales y negras y los desechos de las casas aledañas, lo cual ha generado brotes de plagas y malos olores. La carretera que va desde la UTN hasta Calle Ancha y el centro de Villa Bonita es otro punto crítico que presenta afectación por inundaciones ligadas al ineficiente alcantarillado. Las características morfológicas y el crecimiento urbano han favorecido las inundaciones urbanas en Villa Bonita. En este punto concurre una alta densidad poblacional, rápidos cambios en el uso de la tierra y la falta de planificación, lo que facilitó el aumento del riesgo a lo largo de las últimas décadas (Figura 8).

Figura 8. Centro de Villa Bonita, distrito San Antonio

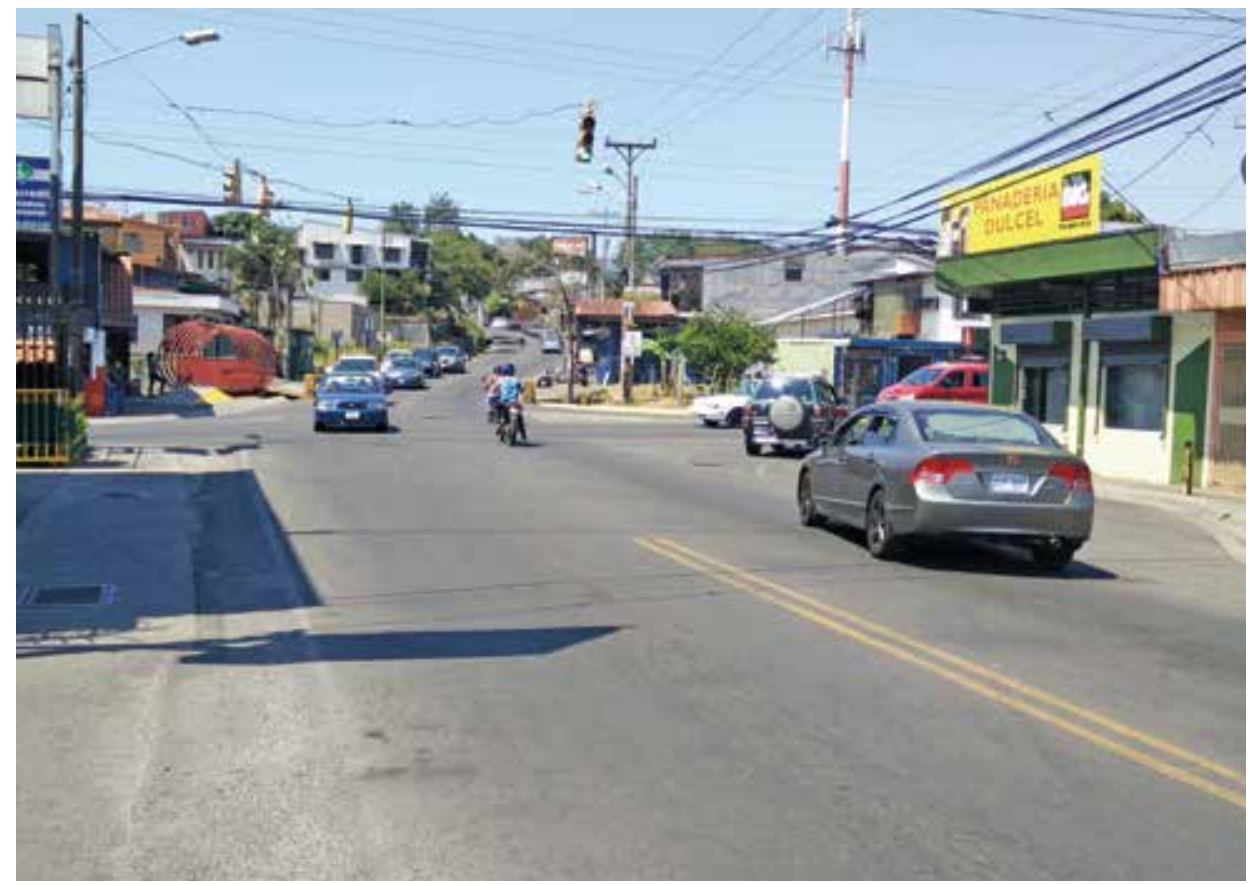

Fuente: Adolfo Quesada Román, 9 de abril de 2016. 
Gustavo Barrantes-Castillo, Adolfo Quesada-Román, Daniela Campos-Durán, Katherine Padilla-Umaña. Indicador de afectación por eventos naturales en el cantón de Alajuela, y su relación con la vulnerabilidad comunal

Por su parte, la comunidad de Ciruelas, en la cual se presentan inundaciones frecuentes, se ubica en el parteaguas de dos importantes cuencas hidrográficas; la cuenca del río Ciruelas y la cuenca del río Siquiares. Este río recoge las aguas residenciales e industriales del sector de El Coyol, se desborda en sus márgenes en la ciudad de Ciruelas, donde el nivel del agua ha alcanzado incluso a los cuatro metros en algunos sectores. Además de ello, en Ciruelas existen otras amenazas de índole tecnológico, como tanques de amoniaco que utilizan las empresas Tropigas, Dos Pinos, Pollo Rey; entre otras. Cabe señalar que en el 2010 se dio una fuga de amoniaco en la empresa Tun a Tun, la cual ya no se encuentra operando en el distrito.

\subsection{Caracterización de la vulnerabilidad en distritos priorita- rios del cantón de Alajuela}

Una vez identificada y caracterizada la amenaza en los sitios críticos, se procede a analizar las condiciones de vulnerabilidad en estas localidades. Cabe resaltar que a pesar de la ocurrencia de eventos que han dejado pérdidas en infraestructura, tales como viviendas, carreteras, puentes e incluso vidas humanas, como el caso del terremoto de Cinchona, no se lleva un registro o recuento histórico de eventos por parte de los entes locales de atención de emergencias ni de la CNE. Esta situación contribuye al aumento de la vulnerabilidad, principalmente, de la población que ha inmigrado recientemente y la población más joven.

Este desconocimiento de los eventos históricos ha contribuido al aumento de la exposición, como es el caso de las construcciones sobre la carretera principal que comunica el distrito de San Isidro con la ciudad de Alajuela, trayecto que ha sido afectado por deslizamientos desde el año 1970, tal como se constató mediante la entrevista realizada en la ADI de San Isidro, o las crecidas sobre Calle Montenegro en Fraijanes que presentan desde el año 1940, sector que actualmente está ocupado por viviendas.

Por otro lado, mediante trabajo de campo se constató que el crecimiento desordenado y las condiciones socio-económicas de sus habitantes han contribuido en la degradación ambiental, principalmente en los sistemas rivereños, donde los ríos se utilizan para el vertido de aguas negras y residuos sólidos. Otro aspecto que se evidencia es la no aplicación de las regulaciones en materia de uso del suelo, así, por ejemplo, en los sitios visitados, la población se ha asentado en áreas que presentan amenazas 
Gustavo Barrantes-Castillo, Adolfo Quesada-Román, Daniela Campos-Durán,

Katherine Padilla-Umaña. Affectation indicator for natural events in the cantón of

Alajuela, and its relation with the community vulnerability

naturales importantes irrespetando la distancia a los ríos y construyendo sobre laderas con fuertes pendientes. Otro factor que ha propiciado el incremento y frecuencia de las pérdidas, especialmente en áreas urbanas, ha sido la capacidad de los sistemas de alcantarillado público, que ha sido rebasada con el crecimiento el uso urbano.

Se consideró el factor socioeconómico dentro de este estudio, ya que el mismo repercute en la capacidad de recuperación o resiliencia de un determinado grupo social frente a un evento natural. En este sentido, es de esperar que los sectores empobrecidos se constituyen en las poblaciones más vulnerables. De acuerdo con los datos obtenidos en campo, de los 38 sitios visitados, el 39\% posee una condición socioeconómica media, principalmente, en los distritos de Sabanilla, Alajuela y San Antonio, y un 31\% poseen una condición socioeconómica media-baja, los cuales se ubican principalmente en los distritos de San Antonio y Alajuela. Por su parte, las áreas con una condición socioeconómica media-alta corresponden al $22 \%$ de los sitios, áreas de mayor poder adquisitivo que se localizan en el distrito San José, en el sector de El Coyol. Finalmente, el estrato más bajo representa el $8 \%$ de los sitios, y se localizan en los distritos de San Isidro, Sabanilla y Alajuela.

Para caracterizar la vulnerabilidad también se consideró el conocimiento que tienen las comunidades sobre los riesgos a los que están expuestos, las instituciones que han trabajado en ellos y la organización comunal que poseen las comunidades ante la posible afectación por eventos naturales, tal como planes de emergencia, identificación de áreas de riesgo, sitios seguros y la infraestructura con la que cuentan y pueda ser usada como albergue.

De acuerdo con los informantes comunales consultados, de las 15 comunidades que se visitaron, solamente Poasito ha recibido información de amenazas sísmicas y volcánicas, además, han participado en simulacros por parte de la CNE. Con este panorama, las condiciones de vulnerabilidad se agravan ya que los esfuerzos institucionales no han logrado promover un conocimiento y educación en la temática de la gestión del riesgo. Por otro lado, se constató que, en las comunidades visitadas, únicamente Poasito y Laguna cuentan con Comité Local de Emergencias. En éstos últimos dos casos el comité no se encuentra activo, por lo cual la población desconoce de las acciones que deben emprender en caso de emergencia o las actividades que debe realizar tendientes a la prevención.

Revista Geográfica de América Central No 59 ISSN 1011-484X, julio-diciembre 2017, pp. 159-196 
Además, se identificó que el $40 \%$ de las comunidades no cuentan con infraestructura que puedan utilizar como alberges en caso de emergencia. En el caso de Poasito, que ha sido una de las comunidades más impactadas por eventos sísmicos e hidrometeorológicos, y San Isidro, afectada mayormente por sismos y deslizamientos, ni siquiera cuenta con un salón comunal, lo cual dificulta las labores de respuesta ante una emergencia.

Con el fin de orientar la priorización de los recursos destinados a la prevención del riesgo, a continuación, se presenta un instrumento que permitió combinar la información cualitativa sobre la amenaza y la vulnerabilidad obtenida a partir de los insumos aportados en este informe. A partir de las fichas de campo, las entrevistas y las inspecciones realizadas se clasificó la amenaza y la vulnerabilidad en tres categorías que respondían a las variables analizadas para establecer estos aspectos. Si bien la valoración realizada es cualitativa el instrumento que se resume en el Cuadro 9permitió la clasificación de los poblados de acuerdo con su nivel de riesgo, resultando San Rafael de Sabanilla como el de mayor riesgo, dada la combinación de condiciones de amenaza y vulnerabilidad presentes en dicho poblado. Le siguen en orden de prioridad Poasito, Fraijanes, San Martín, Barrio San José, la ciudad de Alajuela, Montecillos, Guadalupe y Canoas, Los Higuerones, Montserrat y Villa Bonita. 
Gustavo Barrantes-Castillo, Adolfo Quesada-Román, Daniela Campos-Durán,

Katherine Padilla-Umaña. Affectation indicator for natural events in the cantón of

Alajuela, and its relation with the community vulnerability

Cuadro 9. Valoración cualitativa del nivel de riesgo para la priorización de los esfuerzos en prevención del riesgo.

\begin{tabular}{|c|c|c|c|c|}
\hline Distrito & Poblado & $\begin{array}{l}\text { Valoración de la } \\
\text { amenaza general }\end{array}$ & $\begin{array}{l}\text { Valoración de la } \\
\text { vulnerabilidad } \\
\text { socioeconómica }\end{array}$ & $\begin{array}{c}\text { Valoración } \\
\text { cualitativa } \\
\text { del riesgo }\end{array}$ \\
\hline \multirow{4}{*}{ Sabanilla } & Poasito & 2 & 2 & 4 \\
\hline & Fraijanes & 2 & 2 & 4 \\
\hline & Sabanilla & 1 & 2 & 3 \\
\hline & San Rafael de Sabanilla & 2 & 3 & 5 \\
\hline \multirow{3}{*}{ San Isidro } & San Martin & 2 & 2 & 4 \\
\hline & Pilas & 2 & 1 & 3 \\
\hline & \begin{tabular}{|l|} 
Laguna \\
\end{tabular} & 1 & 2 & 3 \\
\hline \multirow{2}{*}{ San José } & Barrio San José & 2 & 2 & 4 \\
\hline & El Coyol & 2 & 1 & 3 \\
\hline \multirow{4}{*}{ Alajuela } & Alajuela centro & 2 & 2 & 4 \\
\hline & Montecillos & 2 & 2 & 4 \\
\hline & Guadalupe y Canoas & 2 & 2 & 4 \\
\hline & Los Higuerones & 2 & 2 & 4 \\
\hline \multirow{3}{*}{ San Antonio } & Montserrat & 2 & 2 & 4 \\
\hline & Villa Bonita & 2 & 2 & 4 \\
\hline & Ciruelas & 1 & 2 & 3 \\
\hline
\end{tabular}

Fuente: Elaboración propia de los autores.

\section{Conclusiones}

En el cantón de Alajuela las amenazas naturales más recurrentes son las inundaciones y los deslizamientos; no obstante, los terremotos, peligros volcánicos y vendavales tienen una cuota importante de impactos sobre la población. En cuanto a la relación entre las amenazas naturales y la ubicación de los distritos analizados queda claro que los ubicados al norte presentan una predominancia de amenazas volcánicas y sísmicas, los que se encuentran al pie del monte, por deslizamientos e inundaciones rápidas, y los que se encuentran más al sur, en la zona más plana, por inundaciones, principalmente en zonas urbanas.

De acuerdo con el indicador de afectación por eventos desarrollado en esta investigación, en el cantón de Alajuela los distritos de Tambor, la Garita, Turrúcares, Desamparados, la Guácima y San Rafael presentan un

192 Revista Geográfica de América Central No 59 ISSN 1011-484X, julio-diciembre 2017, pp. 159-196 
Gustavo Barrantes-Castillo, Adolfo Quesada-Román, Daniela Campos-Durán, Katherine Padilla-Umaña. Indicador de afectación por eventos naturales en el cantón de Alajuela, y su relación con la vulnerabilidad comunal

grado de afectación histórica menor en comparación con los demás distritos. Por su parte, los distritos de Sarapiquí y Río Segundo presentan un grado de moderado; Sabanilla y San Antonio alto, San Isidro, Alajuela y San José muy alto. Los distritos en los que se concentra este estudio, para la identificación de los sitios críticos de afectación por eventos desastrosos son Alajuela, San José, San Isidro, Sabanilla y San Antonio, dado que su grado de afectación resulta de muy alto a alto.

Los distritos de Sabanilla y San Isidro, ubicados en la ladera SW del volcán Poás, son más propensos a la presencia de deslizamientos, en especial asociados con desprendimientos, caídas, deslizamientos y flujos de lodo. Estos territorios también son susceptibles a inundaciones súbitas que se vinculan con lluvias extraordinarias durante la época lluviosa, relacionadas con sistemas de baja presión o frentes fríos. Los distritos de San José, Alajuela y San Antonio, ubicados en partes más bajas sobre planos y mesetas estructurales, se asocian con una dinámica fluvial de erosión y sedimentación sobre el cauce de los ríos, lo que provoca erosión lateral e inestabilización de puentes. A pesar de no encontrarse llanuras de inundación propiamente, el confinamiento de las quebradas, el mal diseño del alcantarillado, la mala disposición de los desechos sólidos, aguas servidas y negras y el aumento de la escorrentía superficial, a consecuencia de la impermeabilización causada por la expansión urbana, provocan en conjunto frecuentes inundaciones urbanas en estos distritos.

La construcción de viviendas y comercios cercanos a los cauces de los ríos presentan importantes condiciones de vulnerabilidad ante las inundaciones y deslizamientos, lo cual deja en evidencia la falta de controles y del acatamiento de las leyes relacionadas con la planificación urbana. La existencia de puentes antiguos y sin mantenimiento, cuyas estructuras en algunos casos ha sobrepasado su vida útil, representan un elemento expuesto a las crecidas de los ríos. Tal es el caso del puente sobre Quebrada Barro en Montecillos, donde la pérdida de la obra representaría una seria disrupción a las comunidades adyacentes. En los cinco distritos que se visitaron se constató que la red vial es uno de los elementos que más se ha visto afectado por eventos, tales como inundaciones o deslizamientos. La infraestructura crítica que ha sido afectada por inundaciones de manera recurrente, tal como escuelas y colegios, se localiza en el distrito de San José, principalmente en la comunidad del mismo nombre. 
Gustavo Barrantes-Castillo, Adolfo Quesada-Román, Daniela Campos-Durán,

Katherine Padilla-Umaña. Affectation indicator for natural events in the cantón of

Alajuela, and its relation with the community vulnerability

Las comunidades visitadas en los distritos de Alajuela, Sabanilla y San Isidro se caracterizan por presentan una alta vulnerabilidad ante eventos como inundaciones o deslizamientos, como se comprobó mediante trabajo de campo. Por su parte, la población de El Coyol, en el distrito San José, presenta una menor vulnerabilidad ante inundaciones, ya que las condiciones socioeconómicas de su población son media - alta, lo cual se puede apreciar en el crecimiento urbano asociado a condominios, residenciales y urbanizaciones, por lo que esta comunidad presenta una mayor resiliencia para responder ante daños que se pueden generar a raíz de un evento, tal como una inundación.

De las 15 comunidades que se visitaron, solamente en Poasito, se ha manifestado que recibieron información referente a amenazas sísmicas y volcánicas, inclusive que han participado en simulacros organizados por la CNE. En el resto de puntos críticos hay un desconocimiento de la dinámica de las amenazas y de las acciones a tomar, esto de acuerdo con las entrevistas realizadas, lo cual contribuye a aumentar las condiciones de vulnerabilidad de la población. Aún más, en la mayoría de las comunidades visitadas se indica que no cuentan con Comités Comunales de Emergencia, por lo que no se sienten preparados para actuar adecuadamente durante una emergencia, únicamente, Poasito y Laguna poseen Comités, sin embargo, estos no han tenido un funcionamiento constante. De las 15 comunidades visitadas seis no cuentan con infraestructura que puedan utilizar como alberges en caso de emergencia. En el caso de Poasito que ha sido una de las afectadas por eventos sísmicos e hidrometeorológicos y San Isidro, mayormente por sismos y deslizamientos, no poseen ni siquiera un salón comunal que pueda utilizarse como albergue en caso de emergencia.

El poblado de mayor prioridad de atención en materia de disminución del riesgo, de acuerdo con la evaluación cualitativa realizada, es San Rafael de Sabanilla, dada la combinación particular de las condiciones de amenaza y vulnerabilidad que se encuentran en esta localidad. Le siguen Poasito, Fraijanes, San Martin, Barrio San José, la ciudad de Alajuela, Montecillos, Guadalupe y Canoas, Los Higuerones, Montserrat y Villa Bonita. 
Gustavo Barrantes-Castillo, Adolfo Quesada-Román, Daniela Campos-Durán, Katherine Padilla-Umaña. Indicador de afectación por eventos naturales en el cantón de Alajuela, y su relación con la vulnerabilidad comunal

\section{Agradecimientos}

Los resultados de este trabajo están basados en el convenio entre la Universidad Nacional y la Municipalidad de Alajuela mediante la "Contratación de un compendio de información y la base de datos" 000034-01, ejecutada desde la Escuela de Ciencias Geográficas. Se agradece al Consejo Municipal de Alajuela y a su alcaldía por el financiamiento de este estudio y la autorización para la publicación de los principales resultados. Así mismo, se agradece a los miembros de las Asociaciones de Desarrollo Integral por su colaboración e información brindada para esta investigación, Se aclara que la información utilizada en este artículo es propiedad de la Municipalidad de Alajuela.

\section{Referencias}

Alfaro, E., Quesada-Román, A. y Solano, F. J. (2010). Análisis del impacto en Costa Rica de los ciclones tropicales ocurridos en el Mar Caribe desde 1968 al 2007. Revista Diálogos, 11(1), 27-38.

Barrantes, G., Jiménez, C. y Ocón, M.J. (2013). Deslizamientos provocados por el terremoto de Cinchona de 2009, Costa Rica. Revista Geográfica de América Central, 51: 69-79.

Barrantes, G. y Malavassi, E. (2015). Mapa de peligros del volcán Poás. Cuadernos de Geografia: Revista Colombiana de Geografia, 2(24), 157-172.

Barrantes, G. y Salcedo, E. (2016). Consideraciones de la amenaza sísmica en el ordenamiento territorial de cantón de Poás, Costa Rica. Boletín de Geología, 3(38), 109-127.

Comisión Nacional de Prevención de Riesgos y Atención de Emergencias (2009). Evento: sismo 6.2 Richter. Inmediaciones de Cinchona - Poasito - Varablanca Provincia de Alajuela-Heredia. Informe de situación No. 12. Recuperado de https://www.cne.go.cr/web_emer_ cinchona/doc/Informes_CIA/Informe_N12_14_ene_09.pdf

Comisión Nacional de Prevención de Riesgos y Atención de Emergencias - CNE (2015). Amenazas de origen natural en el cantón de Alajuela. Recuperado de www.cne.go.cr

Comisión Nacional de Prevención de Riesgos y Atención de Emergencias - CNE (2009). Decreto No 34993-MP: Alcance N² a la Gaceta $N^{\circ} 9$. Recuperado de https://www.cne.go.cr/Documentos/planes_grales emergencia/CINCHONA.pdf 
Gustavo Barrantes-Castillo, Adolfo Quesada-Román, Daniela Campos-Durán,

Katherine Padilla-Umaña. Affectation indicator for natural events in the cantón of

Alajuela, and its relation with the community vulnerability

Centro Centroamericano de Población, Universidad de Costa Rica (CCPUCR) (2014) InfoCensos. (29 de abril de 2016). Recuperado de http://infocensos.ccp.ucr.ac.cr

LA RED (Red de Estudios Sociales en Prevención de Desastres en América Latina) - Corporación OSSO. (2015). DesInventar: Sistema de inventario de efectos de desastres de Costa Rica entre 1970 y 2015. Ciudad de Panamá, Panamá.

Ley de Planificación Urbana, No. 4240, San José, Costa Rica

Ley Forestal, No 7575.

Ley de Igualdad de oportunidades para las personas con discapacidad, No 7600

Ley Nacional de Emergencias y Prevención del Riesgo, No. 8488

Red Sismológica Nacional: UCR -ICE. (2009). El terremoto de Cinchona del 8 de enero de 2009. Recuperado de http://rsn.ucr.ac.cr/images/ Biblioteca/Informes_sismos/terremoto_cinchona.pdf

Quesada-Román, A. (2015). Implicaciones en la gestión del riesgo de desastres y ambiente en el Valle Central en los últimos treinta años (1985-2015). Vigesimoprimer Informe Estado de la Nación en Desarrollo Humano Sostenible (2014). 